\title{
High Speed PMSM Anti-saturation Regulation Method Based on Hybrid Flux Weakening Technology
}

\author{
$\mathrm{Na} \mathrm{Li}^{1}$, Xiaodong $\mathrm{Ca}^{2 *}$ \\ ${ }^{1}$ JiangSu Polytechnic College of Agriculture and Forestry, Zhenjiang 212400 China \\ ${ }^{2}$ State Grid Jiangsu Electric Power co. LTD, Nanjing 210000 China
}

\begin{abstract}
For traditional permanent magnet synchronous motor (PMSM) flux weakening method, it is easy to cause voltage command saturation and the system lose control. In order to solve this problem, we propose a high speed PMSM anti-saturation regulation method based on hybrid flux weakening technology. The feedforward channel of this method is designed as a two-dimensional table of torque/flux linkage. The expected value of the current current of $\mathrm{dq}$ axis is directly checked through the current flux linkage and torque instruction to ensure the dynamic response of the system. In the feedback channel, the voltage instruction compensation link is proposed in this paper. In combination with the output instruction of the current controller, the speed and bus voltage information, the flux instruction required by the feedforward channel is adjusted. Finally, the experimental results verify that the proposed method meets the requirements of high-speed PMSM fast dynamic response, and improves the reliability of the system when it is running at high speed.
\end{abstract}

\section{Introduction}

Reliability and acceleration performance at high speed are important indexes to evaluate the power performance of electric vehicles. Therefore, the drive motor carried by electric vehicle needs to be able to operate in a wide range of rotational speed. As permanent magnet synchronous motor (PMSM) adopts permanent magnet excitation, it is impossible to adjust the excitation field through the excitation winding. The drive system of PMSM must adopt weak magnetic control technology to meet the speed regulation requirements. However, for traditional PMSM weak magnetic control method, it is easy to cause voltage command saturation and the system lose control. In order to solve this problem, we propose a high speed PMSM anti-saturation regulation method based on hybrid flux weakening technology. The feedforward channel of this method is designed as a twodimensional table of torque/flux linkage. The expected value of the current current of dq axis is directly checked through the current flux linkage and torque instruction to ensure the dynamic response of the system. In the feedback channel, the voltage instruction compensation link is proposed in this paper. In combination with the output instruction of the current controller, the speed and bus voltage information, the flux instruction required by the feedforward channel is adjusted. Finally, the experimental results verify that the proposed method meets the requirements of high-speed PMSM fast dynamic response, and improves the reliability of the system when it is running at high speed.

\section{PMSM driven system modeling}

The structure and mathematical model of PMSM system are shown in figure 1. For the three-phase ideal symmetric PMSM system, the voltage equation of PMSM in the rotating $\mathrm{dq}$ coordinate system is established regardless of the influence of stator resistance.

$$
\left\{\begin{array}{l}
u_{d}=R_{s} i_{d}-\omega L_{q} i_{q} \\
u_{q}=R_{s} i_{q}+\omega L_{d} i_{d}+\omega \psi_{f}
\end{array}\right.
$$

When the PMSM is running at high speed, the stator resistance voltage drop is ignored, and the steady-state current equation of the maximum stator voltage is

$$
\left(-\omega L_{q} i_{q}\right)^{2}+\left(\omega L_{d} i_{d}+\omega \psi_{f}\right)^{2}=\left|u_{s}\right|^{2} \leq V_{s \max }^{2}
$$

The mathematical derivation of equation (2) is carried out and further obtained from the knowledge of analytic geometry

$$
\left(\frac{i_{q}}{a}\right)^{2}+\left(\frac{i_{d}+\psi_{f} / L_{d}}{b}\right)^{2} \leq 1
$$

Where $a=V_{\text {smax }} / \omega L_{\mathrm{q}}$ and $b=V_{\text {smax }} / \omega L_{\mathrm{d}}$.

From the above equation, it can be known that when the PMSM speed runs steadily, the trajectory of its voltage constraint in the $\mathrm{dq}$ coordinate system is an ellipse. Since $\mathrm{Ld}<\mathrm{Lq}$, it can be concluded that $\mathrm{a}<\mathrm{b}$, then the eccentricity of the elliptic trajectory is

$$
e=\frac{\sqrt{b^{2}-a^{2}}}{b}=\frac{\sqrt{\left(V_{s \max } / \omega L_{\mathrm{d}}\right)^{2}-\left(V_{s \max } / \omega L_{\mathrm{q}}\right)^{2}}}{V_{s \max } / \omega L_{\mathrm{d}}}=\sqrt{1-\left(\frac{L_{\mathrm{d}}}{L_{\mathrm{q}}}\right)^{2}}
$$




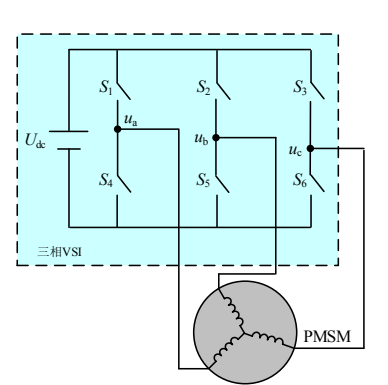

(a) PMSM系统结构图

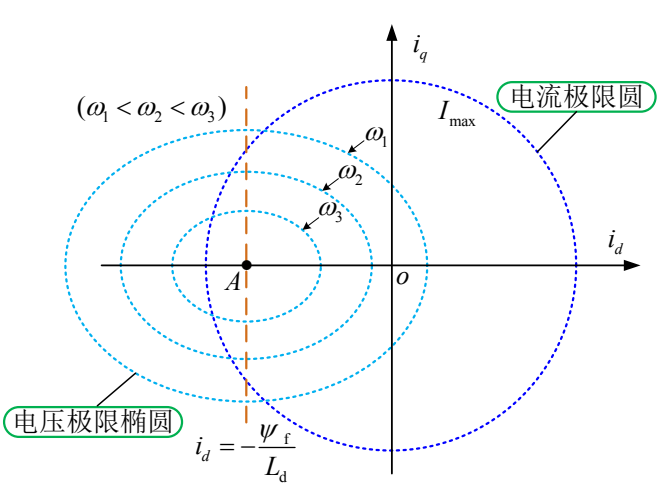

(c) 电压电流限制

Figure 1. The PMSM system structure and mathematical model.

Figure 1(c) shows the voltage limit ellipse corresponding to different operating speeds. We can see that the ellipse obtained from equation (4) is a cluster of nested ellipses with A point $\left(-\psi_{\mathrm{f}} / L_{\mathrm{d}}, 0\right)$ as the center of the circle. When the maximum voltage of the stator is fixed, the voltage ellipse keeps contracting inward with the increase of rotating speed. At the same time, according to the eccentricity formula of equation (2), the shape of the voltage ellipse is closely related to the salient pole $\rho=L_{\mathrm{q}} / L_{\mathrm{d}}$ of the PMSM.

\section{Flux weakening trajectory analysis}

Assuming that PMSM runs in a stable state and its resistance voltage drop is ignored, the flux linkage equation of the motor in dq axis is as follows

$$
\left\{\begin{array}{l}
\varphi_{q}=L_{q} i_{q}=\rho L_{d} i_{q} \\
\varphi_{d}=L_{d} i_{d}+\varphi_{f}
\end{array}\right.
$$

According to formula (5), the flux of motor dq-axis $\psi_{d q}$ can be equivalent to a function of d-axis current.

$$
\left(\varphi_{d q}\right)^{2}=\frac{\left(\rho L_{d} T_{e}\right)^{2}}{\left[\varphi_{f}-(\rho-1) L_{d} i_{d}\right]^{2}}+\left(\varphi_{f}+L_{d} i_{d}\right)
$$

From equation (6), we can see that the motor flux $\psi_{d q}$ will increase with the increase of $\mathrm{d}$-axis current, and when the $\mathrm{d}$-axis current reaches $\psi_{f} /(p-1) L_{\mathrm{d}}$, the motor flux reaches infinity. On the equal torque curve, we can obtain the minimum flux point by partial differentiation of equation (6).

$$
\frac{d\left(\varphi_{d q}\right)^{2}}{d i_{d}}=\frac{2 \rho^{2} L_{d}^{3} T_{e}^{2}(\rho-1)}{\left[\varphi_{f}-(\rho-1) L_{d} i_{d}\right]^{3}}+2 L_{d}\left(\varphi_{f}+L_{d} i_{d}\right)=0
$$

Figure 2 shows the flux weakening trajectory of PMSM. In order to ensure the motor has the maximum torque output capacity in the full speed range, meeting the wide constant power speed requirements of EV drive system. When the PMSM is running in interval II, flux weakening operation is required. Its working point should be moved from MTPA to MFPT along the equal torque curve, with the minimum flux maintained in the process. PMSM working point will not continue to move along the equal torque curve, but gradually move along the MFPT track to point $\mathrm{C}$ with the increasing speed.

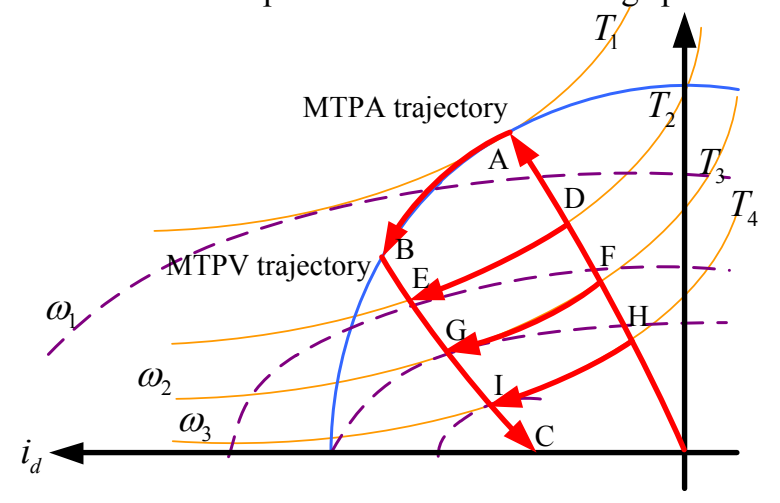

Figure 2. MTPA and MTPV trajectory of PMSM

\section{Hybrid flux weakening technology}

Hybrid flux weakening regulation technology consists of three parts: feedforward path, feedback path and voltage compensation. The above three parts will be designed and analyzed one by one in the following part.

\subsection{Feedforward path design}

When the PMSM running in a flux weakening condition, the flux weakening current can be obtained directly from the given values of torque and flux. At this time, PMSM flux weakening system has high transient response characteristic of magnetic linkage. However, in addition to the parametric dependence, the traditional formula calculation method has not fully considered the optimal trajectory of PMSM weak magnetic movement in the full speed range. In view of the above problems, the feedforward channel adopts the two-dimensional torque/flux linkage look-up method based on formula (7). 

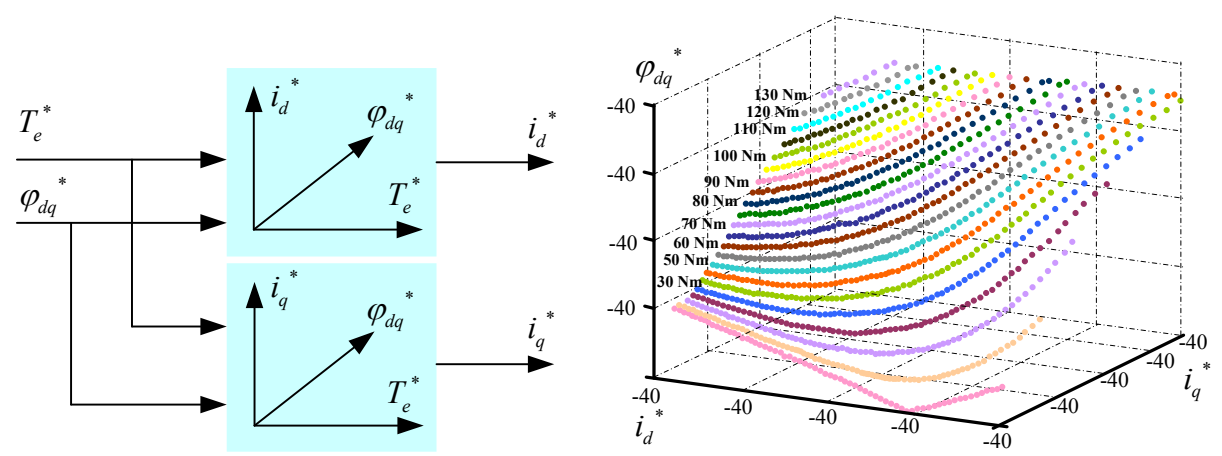

Figure 3. Feedforward channel and two-dimensional lookup table.

\subsection{Feedback path design}

EV usually use lithium battery as the energy source of the electric drive system. When the vehicle is accelerating and regenerative braking, the battery terminal voltage and motor speed will change significantly. Therefore, the design of feedback channel should fully consider motor voltage and speed information. The design structure of the feedback channel is shown in figure 4 . The figure 4 shows that the feedback channel is composed of two modules. The module I calculates maximum amplitude of dq axis flux based on the DC bus voltage $V_{\mathrm{DC}}$ and motor speed $\omega_{\mathrm{e}}$. The Module II calculates amplitude of $\mathrm{dq}$ axis output voltage based on output voltage instructions $u_{\mathrm{d}}{ }^{*}$ and $u_{\mathrm{q}}{ }^{*}$. The deviation between this amplitude and the allowable maximum phase current amplitude is used for PI adjustment to obtain the feedback flux supplementary information $\Delta \psi_{\mathrm{dq}}$.

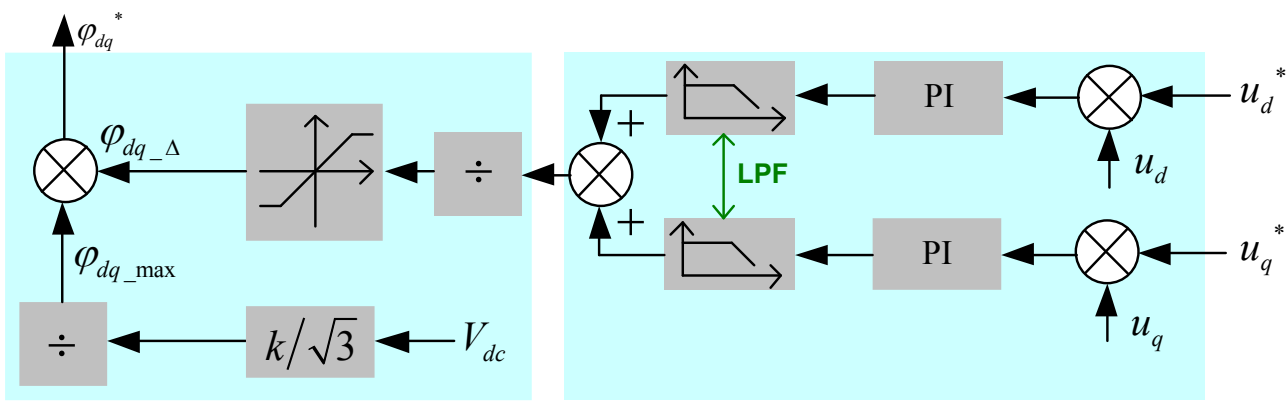

Figure 4. Design structure of feedback channel.

\subsection{Voltage compensation part design}

When PMSM is running at a high speed with flux weakening, the output instruction of the current controller has reached the maximum value set by the inverter. At this time, once the torque is given, the output voltage of the current controller will be saturated, which will affect the output torque capacity of the motor and even cause the whole system to lose control. The output instruction of the inner loop PI regulator before and after compensation is defined as $u_{\mathrm{dq}}$ and $u_{\mathrm{dqc}}$. At the same time, dq axis voltage reverse electromotive force $u_{\text {dqo }}$ is

$$
\left\{\begin{array}{l}
u_{d o}=-\omega_{e} L_{q} i_{q}=-\omega_{e} \varphi_{q} \\
u_{q o}=\omega_{e}\left(\varphi_{f}+L_{d} i_{d}\right)=\omega_{e} \varphi_{d}
\end{array}\right.
$$

When the PMSM running in a flux weakening condition, the motor winding resistance voltage drop is ignored, and the voltage limit elliptic equation is obtained. The essence of the equation is to describe the constraint condition of the electromotive force voltage

$$
\omega_{e} \sqrt{\left(L_{d} i_{d}+\varphi_{f}\right)^{2}+\left(L_{q} i_{q}\right)} \leq u_{o \text { lim }}
$$

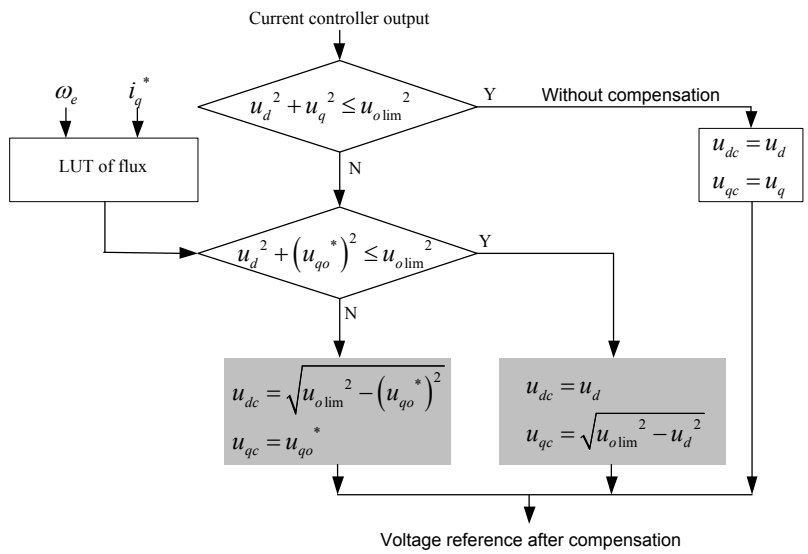

Figure 5. Voltage directive link frame for hybrid flux weakening system.

Figure 5 is the structural block diagram of voltage instruction compensation link. It can be seen whether the output of the current controller exceeds the maximum voltage uolim of the electromotive force. If it does not exceed the maximum voltage uolim, there is no need for correction. The voltage instruction is directly output. If the voltage exceeds uolim, the electromotive force voltage of the $\mathrm{q}$ axis is firstly modified by replacing its original value with the expected electromotive force voltage of the $\mathrm{q}$ axis. If the voltage instruction after 
correction is less than uolim, then the formula in the figure recalcomputes the voltage output instruction of the $\mathrm{q}$ axis. If the voltage instruction after correction is still larger than uolim, the voltage instruction after compensation of axis $\mathrm{q}$ is set as its expected electromotive force, and the voltage instruction after compensation of axis $d$ is obtained according to the formula in the block diagram.

\section{Experimental verification and analysis}

In order to verify the effectiveness of the hybrid flux weakening technology in PMSM system, the steady-state and dynamic performance of the $110 \mathrm{~kW}$ test prototype is experimentally verified.

Figure 6 shows the operation waveform of high-speed PMSM full speed domain based on hybrid weak magnetic regulation. It can be seen from the speed waveform that after the motor is started, the speed rises approximately with a constant acceleration until the given speed. In the case of load disturbance, the speed is almost not affected by it, and the acceleration and deceleration processes are very smooth. Figure 7(a) shows the torque/power-speed characteristic curve of the high-speed permanent magnet synchronous motor. As can be seen from the figure, the actual torque always keeps a constant value below the turning speed $\omega_{\mathrm{cl}}$, and the constant torque amplitude is larger than the constant torque value when the vector method is adopted, indicating that the motor has greater acceleration when the mixed weak magnetic regulation method is applied to start up. Figure 8(b) shows the current trajectory of the high-speed permanent magnet synchronous motor in the full-speed domain under mixed weak magnetic control. It can be seen that, no matter the motor speed is in the running phase of constant torque, running phase of constant power or running phase of reduced power, the actual working point of the motor state has a good tracking effect on the given ideal trajectory. And when the speed is stable and different loads switch with each other, the current state varies along the elliptic trajectory of voltage.

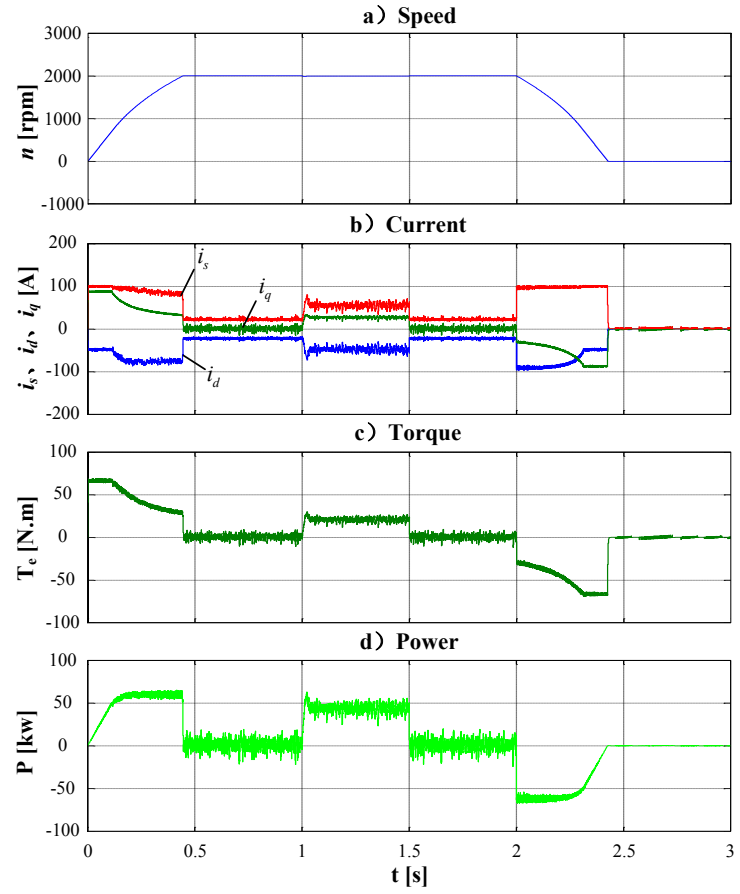

Figure 6. PMSM full speed domain control effect.
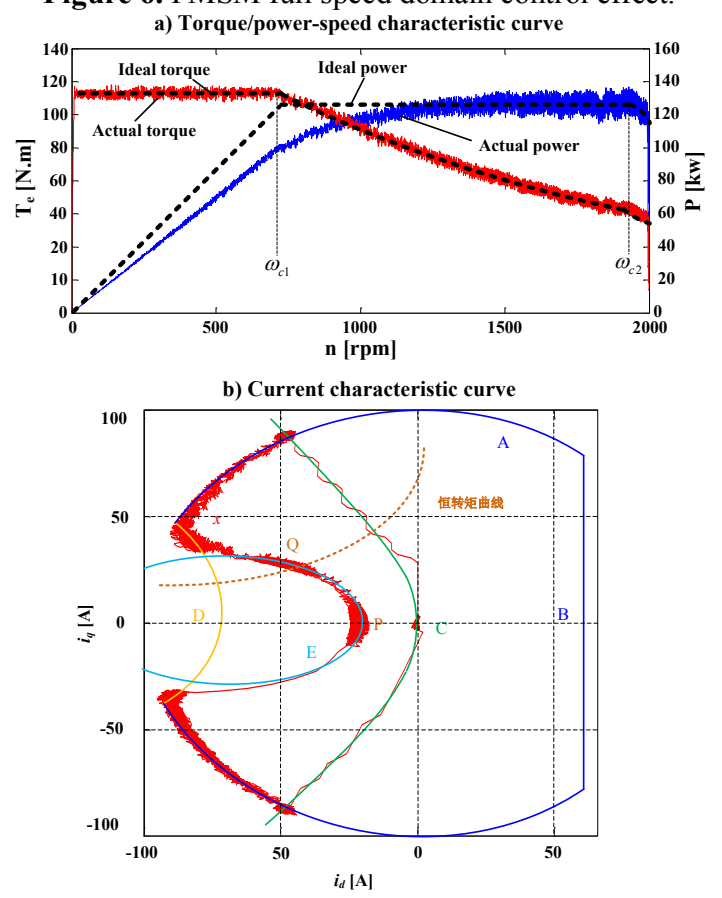

Figure 7. The operation characteristic curve

\section{References}

1. Mazzoletti M A, Bossio G R, Angelo C H D, et al. A Model-Based Strategy for Interturn ShortCircuit Fault Diagnosis in PMSM[J]. IEEE Transactions on Industrial Electronics, 2017, 64(9):7218-7228.

2. Kim J M, Sul S K. Speed control of interior permanent magnet synchronous motor drive for the flux weakening operation[J]. Industry Applications, IEEE Transactions on, 1997, 33(1): 43-48. 
3. Wei W, Feng L, Mei S. Energy Pricing and Dispatch for Smart Grid Retailers Under Demand Response and Market Price Uncertainty[J]. IEEE Transactions on Smart Grid, 2017, 6(3):1364-1374.

4. Jung $\mathrm{S} \mathrm{Y}$, JINSEOK $\mathrm{H}$, KWANGHEE $\mathrm{N}$ A M. Current Minimizing Torque Control of the IPMSM Using Ferrari's Method[J]. IEEE transactions on power electronics, 2013, 28(12): 5603-5617.

5. Feng J, Chu W Q, Zhang Z, et al. Power Electronic Transformer-Based Railway Traction Systems: Challenges and Opportunities[J]. IEEE Journal of Emerging \& Selected Topics in Power Electronics, 2017, 5(3):1237-1253. 